\title{
APPEARANCE OF BIORESORBABLE VASCULAR SCAFFOLDS ON COMPUTED TOMOGRAPHY CORONARY ANGIOGRAPHY
} Singapore Med J 2017; 58(10): 623-625 doi: 10.11622/smedj.2017053

Dear Sir,

Computed tomography coronary angiography (CTCA) is an important imaging technique that has been used for the assessment of coronary artery stenosis. ${ }^{(1)}$ However, its use for the assessment of previous coronary metallic stents is hemmed in by many limitations due to blooming and beam hardening artefacts. ${ }^{(2)}$ With the evolution of coronary stents, the Absorb bioresorbable vascular scaffold (BVS), in which stent material breaks down over time, has become available in clinical practice, hence removing the attendant limitations of metallic implants. ${ }^{(3-7)}$ The availability of the BVS has made CTCA a more appealing modality, and it is prudent that we remain aware of the appearance of bioresorbable scaffolds on CTCA to avoid any misinterpretation.

Based on current appropriate use guidelines, CTCA is considered appropriate for assessing the patency of prior left main coronary stent with stent diameter $\geq 3 \mathrm{~mm}$ in asymptomatic patients. ${ }^{(1)}$ However, it is considered to be an inappropriate test for assessing symptomatic patients with prior unknown coronary stent diameter or with prior stent diameter $<3 \mathrm{~mm}$, while the indication for CTCA is considered uncertain for symptomatic patients with prior coronary stent of diameter $\geq 3 \mathrm{~mm} .{ }^{(1)}$ Nevertheless, these guidelines are presumably based on the fact that the coronary stent is metallic in nature, limiting the accuracy of CTCA for patients who have significant blooming and beam hardening artefacts. ${ }^{(2)}$ In the new era of BVS use in day-to-day clinical practice, CTCA should not be a major issue when evaluating patients with BVS diameter $<3 \mathrm{~mm}$. We herein describe CTCA images from two patients to illustrate the appearance of the BVS and demonstrate that restenosis can be detected without the disadvantages imposed by the metallic struts of non-bioresorbable stents.

Patient 1, a 54-year-old man, had primary percutaneous coronary intervention for acute anterior myocardial infarction in October 2012, in which a BVS $(3.5 \mathrm{~mm} \times 18 \mathrm{~mm})$ was implanted in the proximal left anterior descending artery (LAD). He also had $70 \%$ stenosis in the proximal right coronary artery (RCA). Radionuclide myocardial perfusion imaging done one month later showed no evidence of inducible myocardial ischaemia. He was asymptomatic and had good effort tolerance. Therefore, the RCA lesion was treated medically. CTCA was performed in June 2015 to reassess the RCA stenosis (Fig. 1). CTCA showed patent proximal LAD, with positive remodelling in the segment where the prior BVS was implanted. There was calcified plaque in the proximal RCA, with less than $50 \%$ luminal stenosis. It was important to note the platinum markers at the proximal and distal ends of the previous BVS implantation site. The radiopaque platinum markers had an appearance similar to that of calcified plaques on CTCA.

Patient 2, a 49-year-old man, presented with chest pain and anterior ST segment elevation myocardial infarction in March 2013. Invasive coronary angiography showed $90 \%$ stenosis in the mid LAD and chronic total occlusion in the mid RCA. Primary percutaneous coronary intervention was performed at the LAD with BVSs. The first BVS ( $3.0 \mathrm{~mm} \times 28 \mathrm{~mm})$ was implanted in the mid LAD and a second BVS $(3.5 \mathrm{~mm} \times 18 \mathrm{~mm})$ was deployed proximally, overlapping with the first BVS. Staged percutaneous coronary intervention was performed to the RCA about one month later, when three separate BVSs were implanted in the proximal RCA $(3.5 \mathrm{~mm} \times 28 \mathrm{~mm})$, mid RCA $(3.0 \mathrm{~mm} \times 28 \mathrm{~mm})$ and extending from the mid RCA to the distal RCA $(3.0 \mathrm{~mm} \times 28 \mathrm{~mm})$. The patient was well during follow-up except for occasional vague chest discomfort. Electrocardiography showed new T-wave inversion in leads V2 and V3. In view of this, CTCA was performed in October 2015 and showed occluded LAD with patent RCA. The characteristic

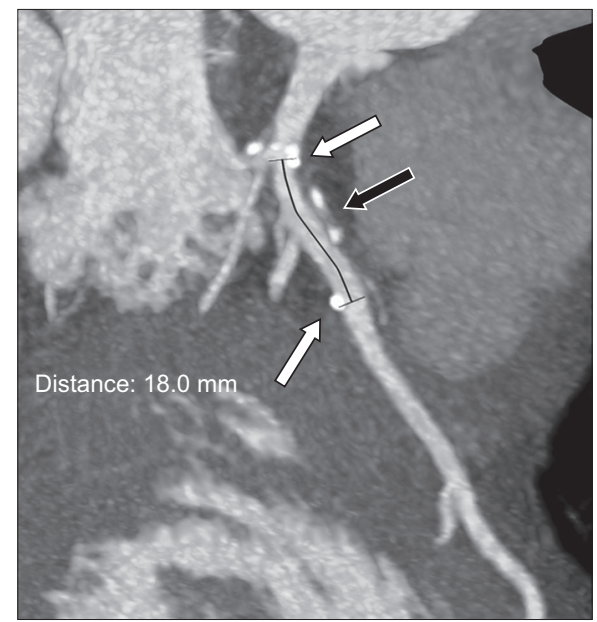

Fig. 1 A 54-year-old man with bioresorbable vascular scaffold (BVS; $3.5 \mathrm{~mm} \times 18 \mathrm{~mm}$ ) implanted in the proximal left anterior descending artery (LAD) for two years and seven months following acute myocardial infarction. CT coronary angiogram under maximal intensity projection (5-mm thickness) shows patent proximal LAD with positive remodelling in the segment where the prior BVS was implanted (black arrow). The prior BVS is not visible. The platinum markers are located at the proximal and distal ends of the previous BVS implantation site (white arrows). The distal left main stem artery appears diseased, with calcified and mixed plaque. 

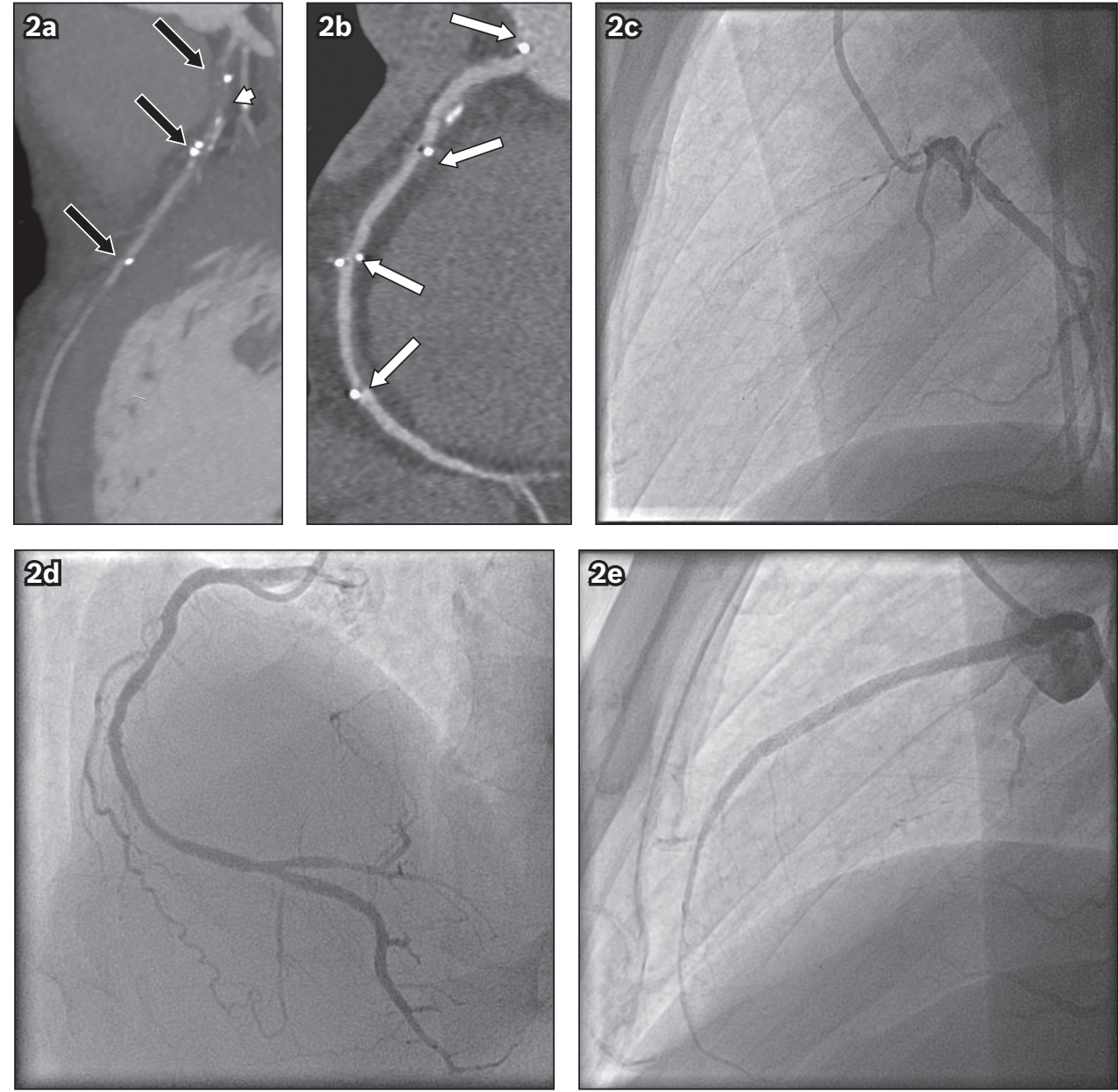

Fig. 2 (a) CT coronary angiogram under maximal intensity projection (5-mm thickness) in a 49-year-old man with two previous Absorb bioresorbable vascular scaffolds (BVSs) implanted in the left anterior descending artery (LAD). The radiopaque platinum markers (black arrows) visualised in the LAD are indicative of two prior BVS implantations. Proximal LAD is occluded with soft plaque (arrowhead). The overlapping portion of the proximal part of the second BVS appears severely diseased with mixed plaque. Beyond the occluded segment, there is contrast filling in the rest of the LAD, suggesting the presence of collateral supply from the other coronary artery. (b) Curved multiplanar reformation of the right coronary artery (RCA) shows the presence of BVS radiopaque platinum markers (arrows), indicative of three prior BVS implantations extending from the proximal RCA to the distal RCA, although they are not visible. There is positive remodelling in the proximal RCA with presence of mixed plaque. Beam hardening is seen at the proximal and distal ends of the prior BVS implantation sites due to the radiopaque platinum markers. Although it might be difficult to exclude the concomitant presence of non-calcified plaque within this small region of artery with beam hardening, the RCA appears patent overall. (c) Invasive left coronary angiogram (87 ${ }^{\circ}$ left anterior oblique [LAO] and $0^{\circ}$ caudal angulation) shows subtotal occlusion of the proximal to mid LAD. (d) Invasive right coronary angiogram (49 LAO and $19^{\circ}$ cranial angulation) shows widely patent RCA providing collateral flow to the occluded LAD. (e) Percutaneous coronary intervention was successfully performed from proximal to distal LAD with three drug-eluting metallic stents.

appearance of the platinum markers was demonstrated again with the overlapping BVS. In addition, the beam hardening artefact was seen (Figs. 2a \& b). Invasive coronary angiogram showed diffuse subtotal occlusion of the proximal-to-mid LAD with collateral flow from RCA, which was patent. Percutaneous coronary intervention was successfully performed to the LAD with three drug-eluting metallic stents $(3.5 \mathrm{~m} \times 38 \mathrm{~mm}, 3.0 \mathrm{~mm} \times 38 \mathrm{~mm}$ and $2.5 \mathrm{~mm} \times 16 \mathrm{~mm}$ ) (Figs. 2c-e).

The two cases presented here underscore the importance of recognising the appearance of platinum markers on CTCA, so as to avoid any misinterpretation of platinum markers as calcified plaques in patients who had a previous BVS. Another advantage of using the CTCA with the non-metallic BVS is that without the risk of blooming and beam hardening artefacts in the stented segment, the CTCA can be used for the evaluation of restenosis in patients with prior BVS implantations. However, at the proximal and distal ends of the BVS, the platinum markers might still give rise to a small region of blooming and beam hardening artefacts. This is especially so if there are multiple overlapping BVSs. Clinicians can use the fact that the platinum markers appear to be located opposite each other at the proximal and distal ends of the BVS to identify them, and this can typically be visualised under maximal intensity projection. This information is essential and might be helpful in differentiating calcified plaques from platinum markers, especially when a prior history of coronary intervention is not available. It should be noted that the inclusion of a past history of previous BVS use is vital to the personnel interpreting the computed tomography images whenever CTCA is ordered.

In summary, caution needs to be exercised when evaluating CTCA in patients with prior BVS implantation to avoid misinterpretation of radiopaque platinum markers as calcified plaques. Radiopaque platinum markers can similarly cause the blooming and beam hardening artefacts seen on CTCA. Overall, the use of CTCA for the assessment of patients with prior BVS implantation should be 
considered analogous to that among patients without prior coronary metallic stents. Current and appropriate guidelines on CTCA use should incorporate indications pertinent to its use among patients with prior BVSs.

Yours sincerely,

Siong Sung Wong ${ }^{1}$, Gunasekaran Ramsamy ${ }^{1}$

${ }^{1}$ Department of Cardiology, National University Hospital, Singapore. siongsung@gmail.com

\section{References}

1. Taylor AJ, Cerqueira M, Hodgson JM, et al. ACCF/SCCT/ACR/AHA/ASE/ASNC/NASCI/SCAI/SCMR 2010 appropriate use criteria for cardiac computed tomography. A report of the American College of Cardiology Foundation Appropriate Use Criteria Task Force, the Society of Cardiovascular Computed Tomography, the American College of Radiology, the American Heart Association, the American Society of Echocardiography, the American Society of Nuclear Cardiology, the North American Society for Cardiovascular Imaging, the Society for Cardiovascular Angiography and Interventions, and the Society for Cardiovascular Magnetic Resonance. J Am Coll Cardiol 2010; 56:1864-94.

2. Hoffmann U, Ferencik M, Cury RC, Pena AJ. Coronary CT angiography. J Nucl Med 2006; 47:797-806.

3. Serruys PW, Ormiston JA, Onuma Y, et al. A bioabsorbable everolimus-eluting coronary stent system (ABSORB): 2-year outcomes and results from multiple imaging methods. Lancet 2009; 373:897-910.

4. Widimsky P, Petr R, Tousek P, et al. One-year clinical and computed tomography angiographic outcomes after bioresorbable vascular scaffold implantation during primary percutaneous coronary intervention for ST-segment-elevation myocardial infarction: the PRAGUE-19 study. Circ Cardiovasc Interv 2015 ; 8:e02933.

5. Ojeda S, Pan M, Romero M, et al. Outcomes and computed tomography scan follow-up of bioresorbable vascular scaffold for the percutaneous treatment of chronic total coronary artery occlusion. Am J Cardiol 2015; 115:1487-93.

6. Suárez de Lezo J, Martín P, Pan M, et al. Bioresorbable vascular scaffold for the treatment of coronary bifurcation lesions: immediate results and 1-year follow up. Rev Esp Cardiol (Engl Ed) 2016; 69:554-62.

7. Asami M, Aoki J, Serruys PW, et al. Feasibility of 320-row multi-detector computed tomography angiography to assess bioresorbable everolimus-eluting vascular scaffolds. Cardiovasc Interv Ther 2016; 31:96-100 